\title{
CERVICAL VERTEBRAL MALFORMATION IN HORSES - CASE REPORT
}

\author{
MiCHELOTTO, P.V. ${ }^{1}$; SENE, N. ${ }^{2}$; TULLIO, D.M. ${ }^{1}$; CAVALLET, A .C. ${ }^{2}$ \\ ${ }^{1}$ Pontifícia Universidade Católica do Paraná - Brasil; \\ ${ }^{2}$ Veterinarias - Pontifícia Universidade Católica do Paraná.
}

The cervical vertebral malformation can result in reduction of the spinal canal with associated compression of the spinal cord. The spinal cord compression can occur in a persistent or recurrent way, in anyone of the cervical vertebrae. The medullar compression can be caused by the surrounding bones, soft tissue, tendons or ligaments. Protrusion of anyone of these structures, into the spinal canal, causes stenosis of the medullar canal, compressing the spinal cord, resulting in ataxia, weakness and spasticity of all the legs. A 3 year-old male thoroughbred, was taken to the Large Animal Hospital of the Pontifícia Universidade Católica do Paraná-PUCPR. The patient fell down after leaving the starting gate, occurring hiperflexion of the neck. After 20 days, signs of simetrical hindlimb ataxia appeared. The attending veterinarian had treated the horse with NSAIDs, corticosteroid, DMSO, Pyrimethamine and trimethoprimsulfadiazine, for two months. The physical examination revealed a grade III/V, ataxia and weakness, of the hindlimbs, appearing slightly more pronounced in the right side, and a grade I/V ataxia of the frontlimbs. A radiographic study, with plain and contrast techniques, was conducted. The myelography was proceeded using lohexol/15.000 mg. A cervical canal stenosis, comprehending C3-C4, was evidenced in the myelographic study. The pronounced clinical signs and prolonged course of the ilness, accounted for the euthanasia decision. The horse had already raced with success before tha accident. So remained a question about the cervical canal stenosis being the cause of the accident or, the accident exacerbating the problem and developing the clinical signs of spinal cord compression. The myelographic examination proved to be a conclusive diagnostic method, evidencing a previous cervical canal malformation.

Key Words: mielography, equine, malformation, ataxia. 